\title{
Synthesis
}

\section{Drivers, "Slow" Variables, "Fast" Variables, Shocks, and Resilience}

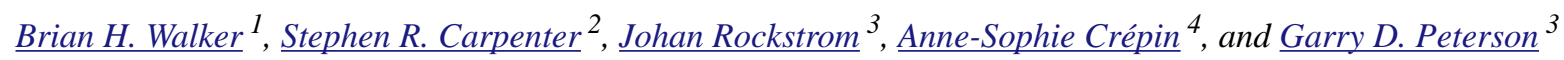

\begin{abstract}
Different uses of the terms "drivers," "variables," and "shocks" cause confusion in the literature and in discussions on the dynamics of ecosystems and social-ecological systems. Three main sources of confusion are unclear definition of the system, unclear definition of the role of people, and confusion between variables and drivers. As a contribution to resolving some of the confusion, we offer one interpretation of how the terms might be used.
\end{abstract}

Key Words: drivers; fast variables; resilience; shocks; slow variables; social-ecological systems

\section{INTRODUCTION}

As everyone's (researchers, managers, society) attention turns to changes in ecosystems and social-ecological systems, we observe with concern a confusing mixture of uses and meanings of terms for fundamental system entities and concepts, in particular with regard to the multiscale behavior of complex adaptive systems. We identify three typical sources of confusion that confound resilience analysis: (1) unclear definition of a social-ecological system; (2) unclear definition of the role of people within the system; and (3) confusion around a system's internal "fast" variables, "slow" variables, "control" variables, and external drivers. We offer the following attempt at clarification, not claiming it is "the" way to use the terms, but as a start toward achieving some agreed understanding.

\section{DISCUSSION}

\section{Sources of Confusion}

1. Confusion due to the lack of definition of the focal "system" most commonly results from people having different, partially articulated problems they wish to address, and therefore, different mental models of what constitutes the real world system (Carpenter et al. 2005). In dynamical terms, a system is defined by (composed of) its state variables, and it is the relationships among them that are of central interest. The system changes as a consequence of both these internal relationships and the effects of external drivers-variables that, within the scale of the analysis, are not considered to be part of the system and are not affected by what happens within the system. Drivers come from higher scales, and one of the sources of confusion in multistakeholder discussions arises when stakeholders have different focal scales, and the focal scale for some includes variables that are "external" for others.

It is necessary to decide what is in and outside the system because this is not always obvious. For example, in a dryland agricultural region, crop production is a state variable that is determined (controlled), at least in part, by the amount of rainfall-an external driver. In an irrigated agricultural region, water available for agriculture may be considered by some to be a given, determined externally by a water authority, but depending on the analysis, it could also be considered as an internal variable subject to different demands by different stakeholder groups. Clarifying how it is to be considered is an essential first step in any analysis, and this requires that people clearly articulate both their concept of a system and their criteria for which components they include and where a system boundary is drawn. Such a process is not trivial and usually requires several cycles of problem specification and model building to clarify people's stated and unstated assumptions and goals (Walker et al. 2002).

2. Failure to clarify the role of humans in the system is a common cause of confusion. They are viewed as exogenous factors (drivers) when interest is on ecological processes and as endogenous when the dynamics of interest are at the social-ecological interface; or, when the object of interest is the social dynamic, the ecosystem is then an exogenous constraint. The conventional way of dealing with peoples' effects within a system, especially in the engineering and economics literature, is to identify variables that people can modify to manipulate the system-termed control variables (the "levers" they can pull to achieve certain objectives). Potential control variables in a fishery, for example, could be harvest rate, subsidies to remote communities, number of boats, or mesh size of nets. The people are users of the ecosystem, and the way they use it is determined by some authority. However, if people are considered part of the system, it is necessary to identify which rules people use to decide on the control variables (their strategies to achieve certain objectives), for example, profit or utility maximization 
under some constraints, replicator dynamics where people tend to follow what other people do, punishment strategies, etc. Each strategy with regard to how to choose the level of control variable will produce particular patterns of interaction with the rest of the system, which may then generate different outcomes. It needs to be clear whether people are just users of the system or whether the dynamics of people-changes in their numbers, welfare, distribution, choices, etc.-are also part of the study objectives (endogenous variables). If so, the ways in which the people variables are influenced and modified through feedbacks from the states of the "natural" system variables need to be considered; and they can change, for example through emigration, thereby feeding back in unforeseen ways to changes in the controls.

3. A common cause of confusion is failing to distinguish between external drivers and internal system variables that act to control the dynamics of other system variables. As just described, feedbacks play an essential role in complex systems, and there are no feedbacks from a complex system to external drivers. Resources that can help provide a systems understanding in relation to resilience concepts include The Resilience Alliance workbook (Resilience Alliance 2011), Bennett et al. (2005), Meadows (2008) for a general introduction to systems, and Walker and Salt (2006).

Within a complex system, it is helpful to focus on separating "fast" and "slow" variables (see, e.g., Ludwig et al. 1978, Holling 1986, Carpenter and Turner 2000 for ecosystems; Crépin 2007 for social-ecological systems). "Fast" variables are typically those that are of primary concern to ecosystem users, for example a pest species or (often) ecosystem goods and services, such as crop production, clean water, and favored species. The dynamics of these fast variables are strongly shaped by other system variables that generally change much more slowly, and hence have been referred to as "slow", or (because they are not always slow) "controlling" variables. They are not the same as the control variables mentioned in (2) above, and to avoid confusion, we suggest it is best to simply refer to them as "slow" variables, recognizing that "fast" and "slow" are relative terms. The slow variables, such as amount of soil organic matter, shape how a fast variable, such as crop production, responds to variation in an external driver, such as variation in rainfall during the growing season.

The equilibrium levels of fast variables for given amounts of the slow variables are shaped by their interaction with external drivers. In many situations, the relationship between the fast and slow variables is monotonic (cannot generate mathematical bifurcations), as in Fig. 1a and b, but for some pairs, it is discontinuous, i.e., mathematical bifurcations can occur (Fig. 1c). In a case such as Fig. $1 \mathrm{c}$, there is a hysteresis effect in the return path of the fast variable (FV) as the slow variable (SV) changes back toward its former level, resulting in alternate system regimes-in which the FV can be attracted to different levels, depending upon the system's history. These alternate regimes are separated by a threshold, indicated by the dotted line. Crossing this threshold causes the FV to be attracted toward a different equilibrium level.

Fig. 1. The relationship between the equilibrium amounts of a fast social-ecological good or service (FV) and the slow variable (SV) that controls it. (a) and (b) are (mathematically) linear relationships. (c) is non-linear, resulting in alternative levels of the FV for the same amount of the SV, separated by an unstable threshold (dotted line).

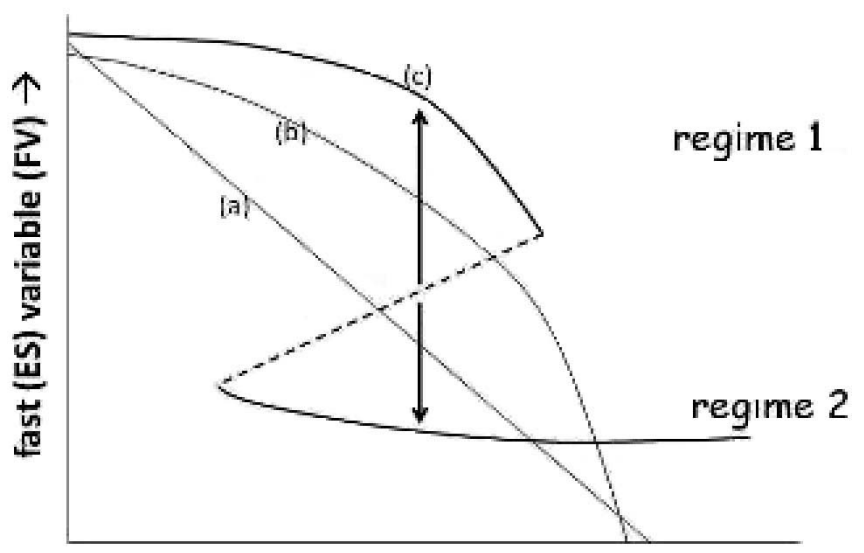

slow (controlling) variable (SV) $\rightarrow$

The levels of the SV are, in turn, determined by drivers external to the system, as depicted in Fig. 2, and sometimes also by other state variables in the system. Confusion arises when people confound the external drivers and internal dynamics that cause the reorganization. Separating internal SVs from external drivers is essential to understanding system dynamics; however, in practice, clearly separating them can be difficult.

The amounts of a FV are not constant. They fluctuate around a long-term attractor (a stable equilibrium state) due to both external shocks to the system (changes in external drivers, commonly environmental variation, or actions by people outside the system) and changes within the system itself, which can change the attractor. However, as long as the amounts of a FV do not cross a threshold level, they tend to remain near or return toward the attractor points. The magnitude of these fluctuations (variance) often increases as the SV approaches a threshold level (Fig. 3) due to an interaction of the external 
Fig. 2. External (or higher scale) drivers cause changes in the slow (controlling) variables (SV), as in part (b), which then in turn determine the amounts of the faster changing system goods and services (FV), as in part (a)

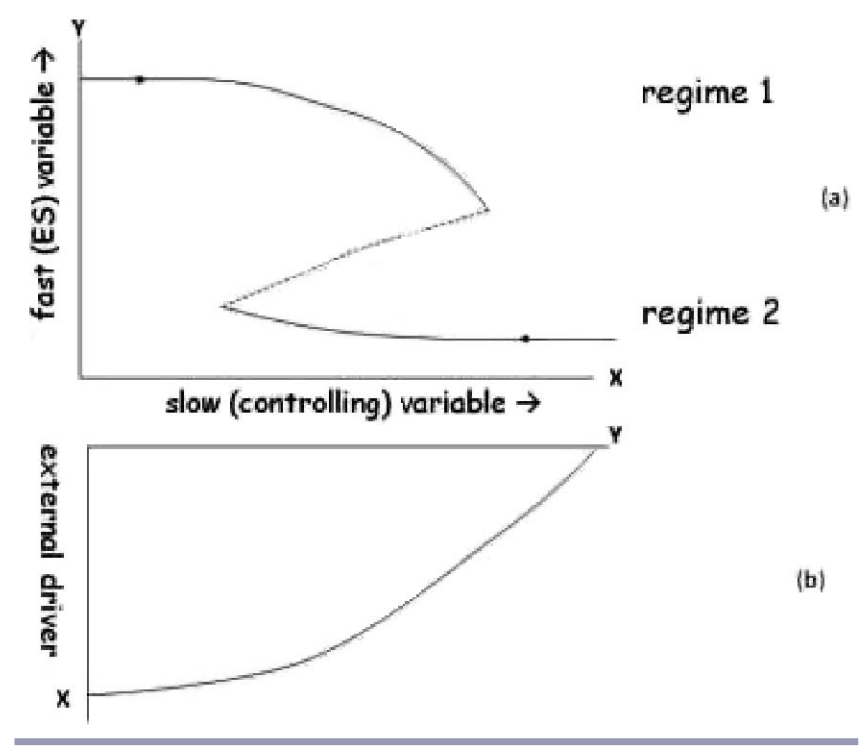

shocks with the internal dynamics. This phenomenon, known as rising variance, occurs because of a weakening of stabilizing feedback loops as the system approaches a threshold, and has been used as an early warning indicator of an approaching threshold (Carpenter and Brock 2006, Scheffer et al. 2009).

The interactions between internal dynamics and external drivers can be quite complex. Because of internal dynamics, a system is more likely to reorganize in response to external drivers than just react passively. However, whether it actually experiences a reorganization depends upon the sequence of shocks it experiences. For example, a forest may become more combustible, but if there is no fire ignition event, it will not burn. Conversely, changes in a driver can change the frequency and intensity of shocks that a system experiences. For example, a shift in climate can alter the frequency of thunderstorms that produce fire initiation events as well as the combustibility of a forest. Because changes in frequency can only be observed over times longer than the frequency, they can be difficult to detect. However, having a systemic understanding of how external drivers interact with internal dynamics can help identify possible alternate system configurations.

\section{CONCLUSION}

The short message from this note is: Drivers (external to the system, or from higher scales) cause change in "slow" (controlling) variables; as slow variables approach threshold levels, the fast-moving variables in the system fluctuate more in response to environmental and other shocks; and these shocks or directional change in the drivers can push the system across a threshold into an alternate stability regime.

Fig. 3. The variance around the stable state of a fast variable (good/service) rises as the slow variable approaches a threshold level, due to an interaction of exogenous variance and internal ecosystem processes. The fluctuations become larger at the same time resilience is becoming smaller. Such "shocks" to the fast variable can push it across the threshold and cause the system to shift into the alternative regime (stability domain).

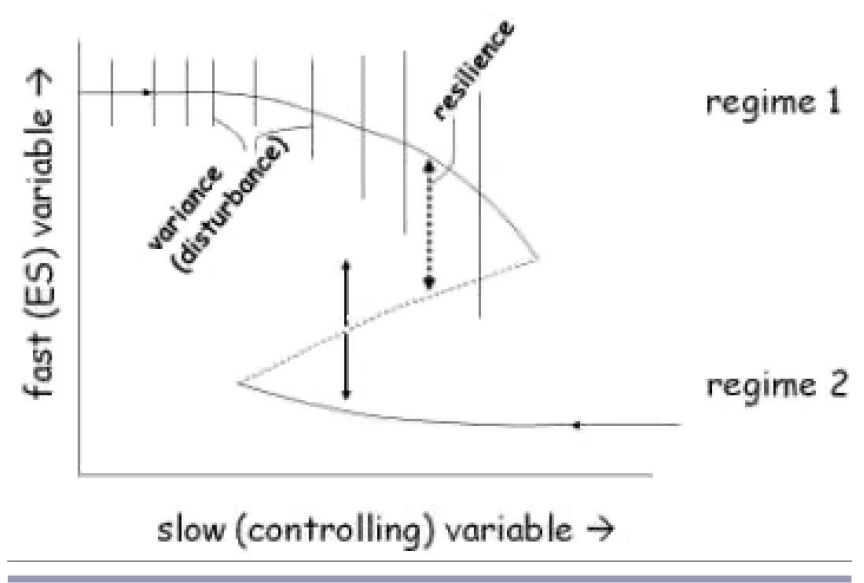

To steer such a system in a desired direction by manipulating the control variables, while at the same time avoiding nasty surprises, it is necessary to take into account not only the fast variables' dynamics, but also (especially) the dynamics of their relevant controlling (slow) variables together with any threshold effects they might have, and how these slow variables respond to external drivers.

Responses to this article can be read online at: http://www.ecologyandsociety.org/voll7/iss3/art30/ responses/

\section{Acknowledgments:}

We thank Eddy Carmack and Annika Nilsson for initial comments on problems with using these terms, and two referees for their very useful comments.

\section{LITERATURE CITED}

Bennett, E. M., G. S Cumming, and G. D. Peterson. 2005. A systems model approach to determining resilience surrogates for case studies. Ecosystems 8:945-957. 
Carpenter, S. R., and W. A. Brock. 2006. Rising variance: a leading indicator of ecological transition. Ecology Letters 9:311-318. http://dx.doi.org/10.1111/j.1461-0248.2005.00877. $\underline{\mathrm{x}}$

Carpenter, S. R., and M. G. Turner, editors. 2000. Special issue on interactions of fast and slow variables in ecosystems. Ecosystems 3(6):495-595. http://dx.doi.org/http://dx.doi.org/ $\underline{10.1007 / \mathrm{s} 100210000043}$

Carpenter, S. R., F. Westley, and M. G. Turner. 2005. Surrogates for resilience of social-ecological systems. Ecosystems 8:941-944 http://dx.doi.org/10.1007/s10021-005 $\underline{-0170-\mathrm{y}}$

Crépin, A.-S. 2007. Using fast and slow processes to manage resources with thresholds. Environmental and Resource Economics 36:191-213. http://dx.doi.org/10.1007/s10640-00 6-9029-8

Holling, C. S. 1986. The resilience of terrestrial ecosystems: local surprise and global change. Pages 292-317 in W. C. Clark and R. E. Munn, editors. Sustainable development of the biosphere. Cambridge University Press, Cambridge, UK.

Ludwig, D., D. Jones, and C. S. Holling. 1978. Qualitative analysis of insect outbreak systems: the spruce budworm and forest. Journal of Animal Ecology 47:315-332. http://dx.doi.o rg/10.2307/3939

Meadows, D. H. 2008. Thinking in systems—a primer. Chelsea Green Publishing, White River Junction, Vermont, USA.

Resilience Alliance. 2011. Assessing resilience in socialecological systems: workbook for practitioners. Version 2.0. [online] URL: http://www.resalliance.org/3871.php

Scheffer, M., J. Bascompte, W. A. Brock, V. Brovkin, S. R. Carpenter, V. Dakos, H. Held, E. H. van Nes, M. Rietkerk, and G. Sugihara. 2009. Early-warning signals for critical transitions. Nature 53:53-59. http://dx.doi.org/10.1038/nature $\underline{08227}$

Walker, B. H., S. Carpenter, J. M. Anderies, N. Abel, C. Cumming, M. Janssen, L. Lebel, J. Norberg, G. D. Peterson, and R. Pritchard. 2002. Resilience management in socialecological systems: a working hypothesis for a participatory approach. Conservation Ecology 6(1): 14. [online] URL: http ://www.consecol.org/vol6/iss1/art14

Walker, B. H., and D. Salt. 2006. Resilience thinking: sustaining ecosystems and people in a changing world. Island Press, Washington, D.C., USA. 